\title{
Method of face recognition from thermal images
}

\author{
by A. Andonova* and A. Radev*
}

*Dept. of Microelectronics, Technical University of Sofia, 8 blvd. KI. Ohridski, 1797 Sofia, Bulgaria, ava@ecad.tu-sofia.bg

\section{Abstract}

The current article examines an approach for face recognition based on thermal imaging methods and Matlab image processing. Images of persons, captured with infrared camera, are submitted to filtration and morphological operations in order to extract parts of the face that could be used for its recognition. Then the images are processed in order to determine the specific contours of the face of the captured person as well as the blood vessels. The aim of this article is to present the methods of image processing and comparison between the images of individuals used in this face recognition system.

\section{Introduction}

Nowadays, face recognition techniques using images in the visible spectrum of light, are widespread. These methods are used in applications for people's tracking and detection and access control systems. Especially important is the face recognition of individuals in systems for detecting terrorists in the dark. In fact, the reliability of the existing face recognition systems is not very high and depends on several factors, most important of which are illumination of the space, distance to camera, facial expression, changing of the face over time and others $[1,2,3]$. Some of these problems may be solved by the means of thermal imaging $[4,5]$. In [1] are described in details the advantages of face recognition using thermal imaging methods compared to the image recognition in the visible spectrum.

In [6] was presented an approach for processing of infrared images that successfully separated the main contours of the face and the blood vessels. It was shown that the area, including the eyes and a certain perimeter around them, is most suitable for use in face recognition systems, since it is slightly affected by the change of expression and other confounding factors. The blood vessels and the contours, located in this area, are used in the current work for recognizing individuals.

\section{Determination of the area of interest}

It was already mention that it is necessary to separate an area around the eyes of the original image, which carry the information for the description and recognition of the person.

It was found by experience that the both ends of the eyes, which are close to each other, are distinguished by relatively high temperature compared to other parts of the face. The initial image processing involves the application of an image conversion, based on threshold for determining the areas of the face with the highest temperature. By applying morphological operations, each of these areas shrinks to one point, as shown in the figure1.

An algorithm for comparison between these points and the determination of those two, which are most likely to indicate the location of the eyes, has been developed. This algorithm is applied for every two points at close vertical level. The temperature of the area between every two points is monitored, as well as their location on the image.
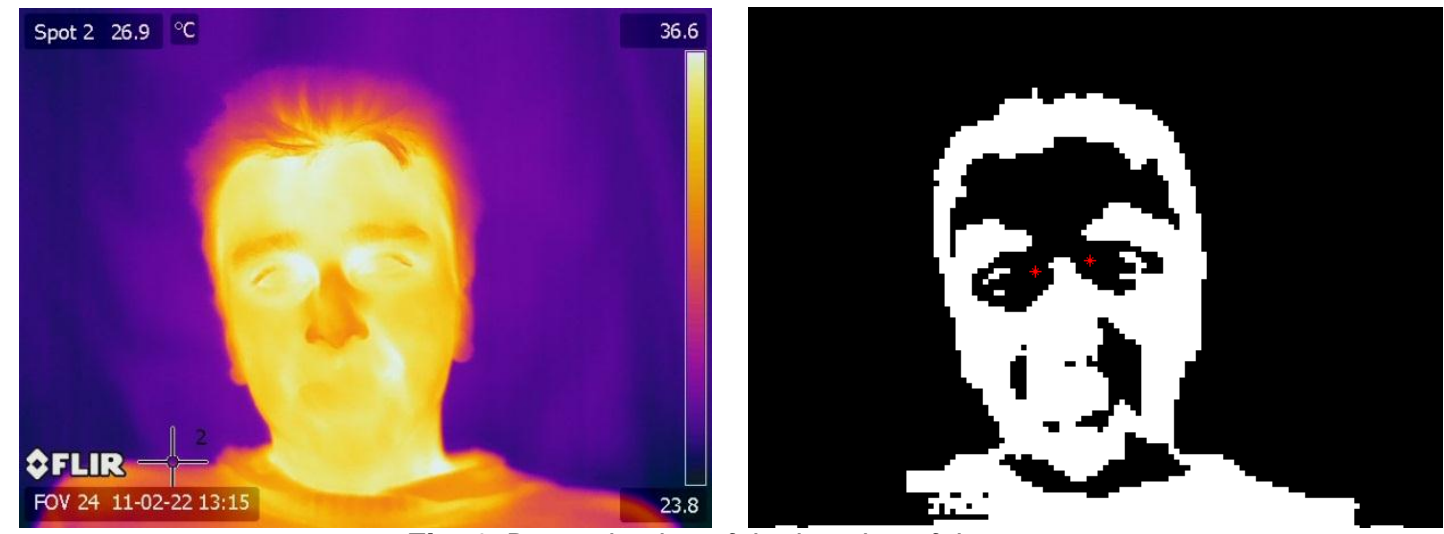

Fig. 1. Determination of the location of the eyes 
Further, the area around the two points that are most likely to indicate the eyes is cut from the original image. The size of this area depends on the distance between the points - thus a change of the distance between the camera and individual will result in area with different number of pixels, but it will capture approximately the same regions of the face for every picture (figure2).
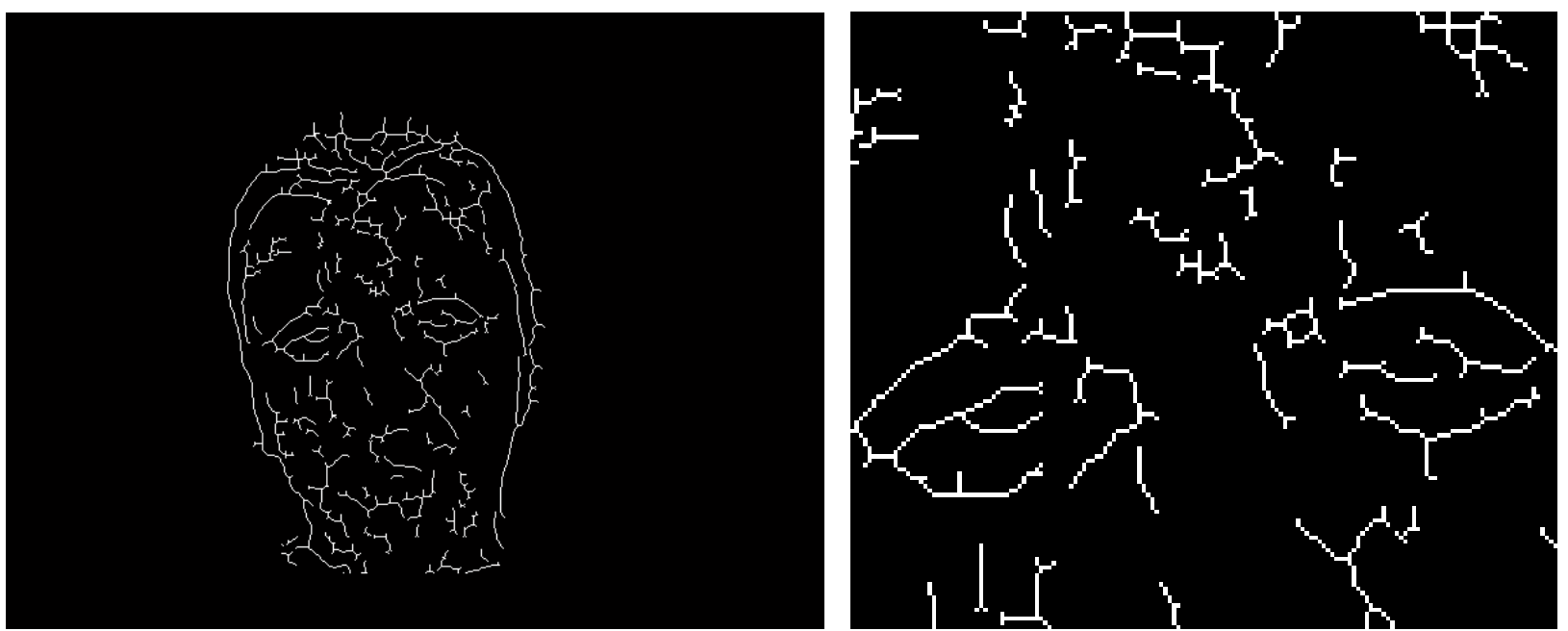

Fig. 2. Contours and blood vessels of the face; contours and blood vessels in the area cropped around the eyes

\section{Further processing}

Further processing is applied to the images as described in [2].

The determination of the contours and the vascular system is done by applying a top-hat filter. This filter gives as result an image that is equal to the difference between the original input image and its morphological opening. The opening of an image on the other hand is a morphological erosion of the image followed by dilation:

$$
\begin{aligned}
& I_{\text {cont } / \text { vessels }}=I_{\text {input }}-I_{\text {opened }} \\
& I_{\text {opened }}=(I \ominus S R) \oplus S R
\end{aligned}
$$

where SR is $3 \times 3$ structuring element and $l_{\text {opened }}$ is the morphological opening of the image, which is defined as erosion followed by dilation, denoted as $\Theta$ and $\oplus$ respectively.

Additional morphological operations for removing the smallest objects, filling the isolated interior pixels and bridging closely located pixels were applied to the images in order to obtain clearer results. Some of these operations are shown in the code below:

$$
\begin{aligned}
& \text { processed_image }=\text { imtophat(processed_image, } m a t r i x 1) \text {; } \\
& \text { processed_image }=\text { im } 2 \mathrm{bw}(\text { processed_image }, 0.00001) \text {; } \\
& \text { processed_image = bwareaopen }(\text { procéssed_image }, 10) \text {; } \\
& \text { processed_image = bwmorph(processed_image,'bridge',Inf); } \\
& \text { processed_image }=\text { bwmorph }(\text { processed_image,'fill'); } \\
& \text { processed_image = bwmorph(processed_image,'close',Inf); } \\
& \text { processed_image }=\text { bwmorph }(\text { processed_image, 'thicken',1); } \\
& \text { processed_image = bwmorph(processed_image,'skel',Inf); } \\
& \text { processed_image = bwareaopen }(\text { processed_image }, 10) \text {; }
\end{aligned}
$$

On each of the resulting lines are placed points that indicate the end of the line or branch, as well as intermediate points (figure3). For each of these points are carried out operations aimed at description of the vicinity of the points and determining their approximate location in the image. 


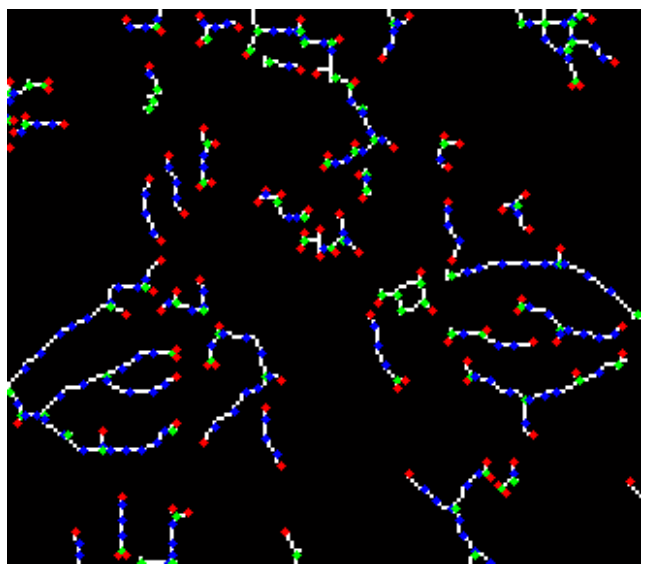

Fig. 3. Placement of points for indication of end (red) and branch (green) of line and intermediate points (blue).

By the description of the vicinity of points, the approximate direction of the blood vessels and the contours could be determined. The vessels and the contours are described with several vectors with selected in advance directions and the size of these vectors is determined by the vicinity of the points that are being examined. It should be pointed that all points in the image are described with the same set of vectors. Thus, different direction of the vessels or the contours will result only in different size of these vectors (figure4).
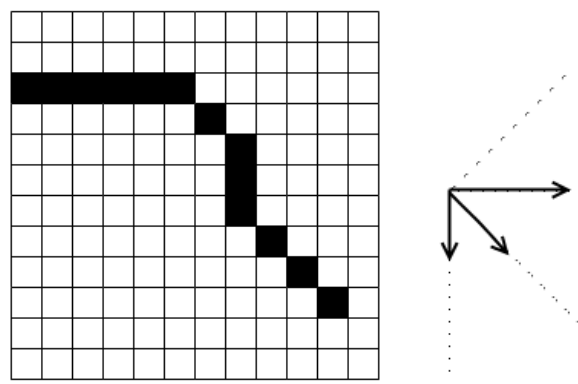

Fig. 4. Description of blood vessel or contour - the line, given as example, is described with the length of four vectors with pre-determined direction

Further the image is automatically divided into regions. The aim of the final processing is to determine the number of points of every type and the dimensions of the vectors used to describe the vessels and the contours for every region. The results are given in tabular form (figure 5) and the recognition process is reduced to comparison of the vectors and the number of points for the same regions in different pictures.

The images were divided into 30 equal regions, but future researches on the dependence between the size and number of the regions and the recognition process will be made.

\begin{tabular}{|r|r|r|r|r|r|r|r|r|}
\hline \multicolumn{1}{|c|}{1} & \multicolumn{1}{|c|}{2} & \multicolumn{1}{l|}{3} & 4 & 5 & 6 & 7 \\
\hline 1 & 1 & 2 & 3 & 4 & 5 & 6 & 7 \\
\hline 2 & 82.3958 & 83.7375 & 83.5264 & 73.4694 & 1.0410 & 8.2535 & 22.3264 \\
\hline 3 & 44.2014 & 45.2292 & 39.4375 & 33.2056 & 0.5028 & 5.9167 & 11.8403 \\
\hline 4 & 87.4653 & 89.9417 & 84.1243 & 74.1535 & 0.9403 & 6.5764 & 23.7847 \\
\hline 5 & 44.1667 & 49.5792 & 50.0250 & 46.6583 & 0.7514 & 10.8125 & 11.9444 \\
\hline 6 & 144 & 144 & 144 & 144 & 144 & 144 & 144 \\
\hline 7 & 1 & 1 & 1 & 1 & 1 & 1 & 1 \\
\hline 8 & 39.5139 & 42.8542 & 39.2292 & 33.8597 & 0.4701 & 6.0660 & 10.6944 \\
\hline 9 & 29.0625 & 31.6000 & 31.7056 & 27.8264 & 0.3715 & 3.3785 & 7.8819 \\
\hline 10 & 58.6111 & 57.6639 & 54.3500 & 51.1194 & 0.8076 & 10.1285 & 15.8333 \\
\hline
\end{tabular}

Fig. 5. The table represents the image after the final processing. Each column corresponds to one region of the image

\section{Initial results and future work}

In the current work a database with 360 thermograms of 120 different persons has been used. The verification of the recognition system was done through the comparison of image of a person, who already exists in the database, with all other images from the database [4]. The initial results show that the system detects similarities between two different pictures of the same person. 
For example, assuming that the comparison of an Image1 with database that already contains Image1 would show $100 \%$ match, the comparison between Image2 (which is not part of the database) and the database results in $82 \%$ match between Image1 and Image2 (figure6). The next closest scores are $61 \%$ and below.
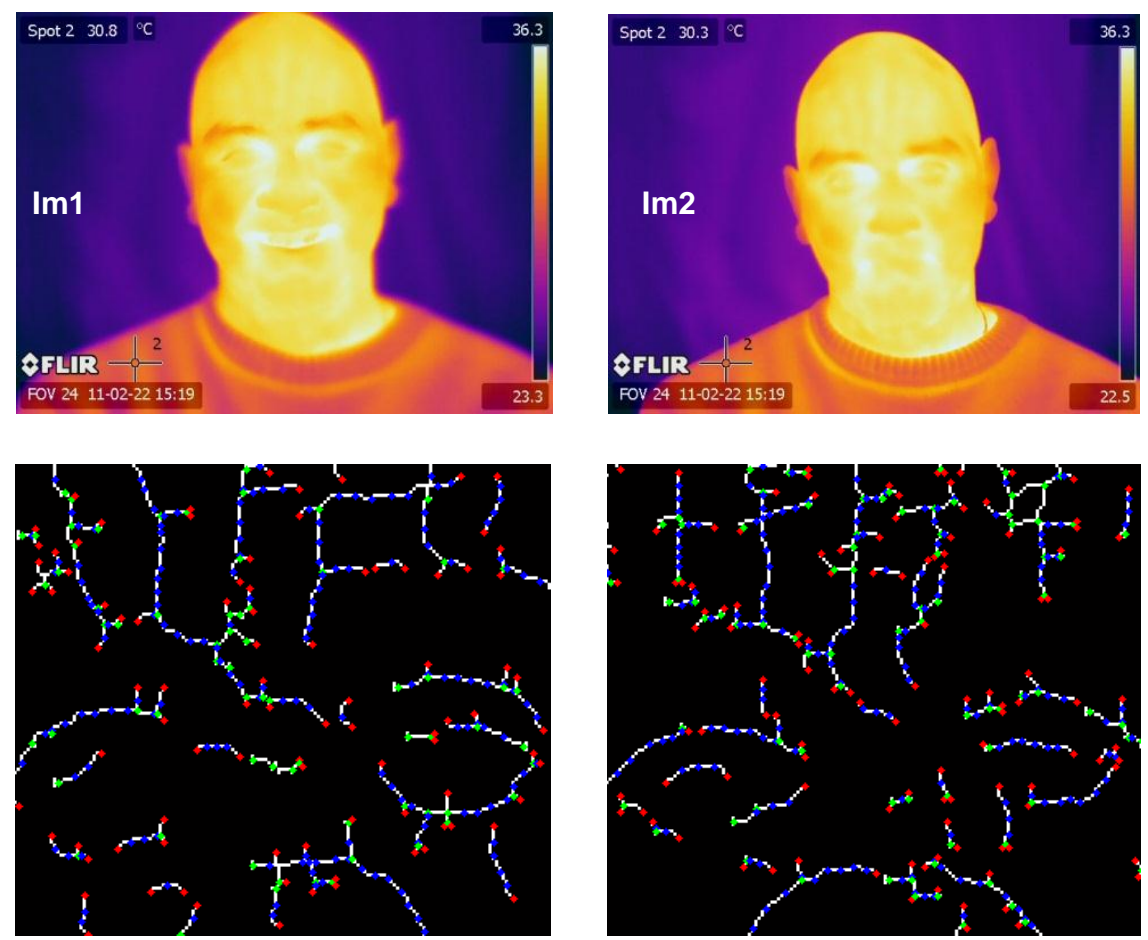

Fig. 6. Contours and blood vessels of individual with different face expression and different temperature of the background

The face recognition system is still under development and there are no strictly defined criteria to determine whether the recognition was successful or not, although the initial results are encouraging. Many statistical researches must be held in order to determine the influence of the distance between the camera and the person, the position of the head on the processing, the number of regions used to split the image. The team is currently working on expansion of the database, researching new criteria that could be used in the face recognition system as well as improvement of the methods for initial processing of the images that have already been presented.

\section{Conclusions}

The current paper presents methods used for processing and description of thermal images of individuals that will be used in face recognition system.

\section{Acknowledgements}

This work was supported by National Ministry of Science and Education of Bulgaria under Contract DDVU 02/47: "Thermo Vision Methods and Recourses in Information Systems for Customs Control and Combating Terrorism Aimed at Detecting and Tracking Objects and People".

\section{REFERENCES}

[1] Reviews, Refinements and New Ideas in Face Recognition, edited by P. M. Corcoran, InTech, Croatia, 2011.

[2] Xie Z., Zeng J., Liu G., Fang Z., "A novel infrared face recognition based on local binary pattern", Proceedings on ICWAPR 2011, pp. 55-59, 2011

[3] Junfeng B., Yong M., Jing L., Fan F., Hongyuan W., "Novel averaging window filter for SIFT in infrared face recognition," Chinese Optics Letters, vol. 9, no 8, pp. 081002-081002, 2011

[4] Xie Z.., Liu G. ," Infrared Face Recognition Based on Local Binary Pattern and Pattern Selection", Journal of Computational Information Systems vol. 7, no 12, 4367-4374, 2011

[5] Xie Z., Guodong L.,"Weighted Local Binary Pattern Infrared Face Recognition Based on Weber's Law”, Proceedings of the Sixth International Conference on Image and Graphics ICIG '11, pp. 429-433, 2011

[6] Radev A., Andonova A., "Face Recognition Using Infrared Images", Annual journal of electronics, vol.5, no2, pp.146-148, 2011 